\title{
Modular System for Probabilistic Fracture Mechanics Analysis of Embrittled Reactor Pressure Vessels in the Grizzly Code
}

B. W. Spencer, W. M. Hoffman, M. L. Backman

October 2018

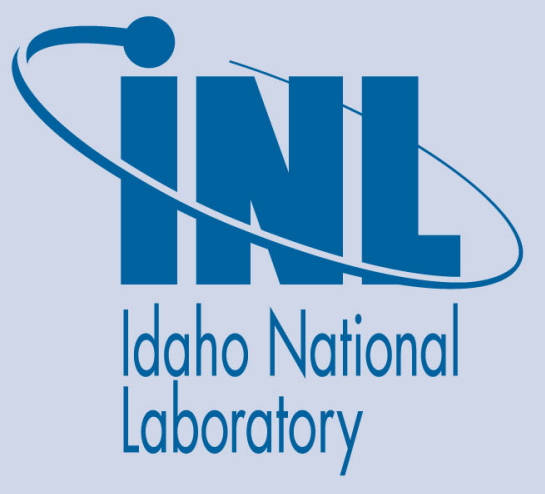

The INL is a U.S. Department of Energy National Laboratory operated by Battelle Energy Alliance 


\title{
Modular System for Probabilistic Fracture Mechanics Analysis of Embrittled Reactor Pressure Vessels in the Grizzly Code
}

\author{
B. W. Spencer, W. M. Hoffman, M. L. Backman
}

October 2018

Idaho National Laboratory Idaho Falls, Idaho 83415

http://www.inl.gov

Prepared for the

U.S. Department of Energy

Under DOE Idaho Operations Office

Contract DE-AC07-05ID14517 


\title{
Modular System for Probabilistic Fracture Mechanics Analysis of Embrittled Reactor Pressure Vessels in the Grizzly Code
}

\author{
B. W. Spencer ${ }^{*}, \mathrm{a}$, W. M. Hoffman ${ }^{\mathrm{b}}$, and M. A. Backman ${ }^{\mathrm{c}}$ \\ ${ }^{a}$ Fuel Modeling and Simulation, Idaho National Laboratory, P.O. Box 1625, Idaho Falls, ID \\ 83415-3840 \\ ${ }^{\mathrm{b}}$ Systems Integration, Idaho National Laboratory, P.O. Box 1625, Idaho Falls, ID 83415-3840 \\ ${ }^{\mathrm{c}}$ Department of Nuclear Engineering, University of Tennessee, 1412 Circle Dr. Knoxville, TN \\ 37996-1410 \\ *Corresponding Author: benjamin.spencer@inl.gov
}

October 5, 2019

\begin{abstract}
This article is published as: B.W. Spencer, W.M Hoffman, and M.A. Backman. Modular system for probabilistic fracture mechanics analysis of embrittled reactor pressure vessels in the Grizzly code. Nuclear Engineering and Design, 341:25-37 (2019). 10.1016/j.nucengdes.2018.10.015
\end{abstract}

\begin{abstract}
In light water reactor (LWR) nuclear power plants, the reactor pressure vessel (RPV) plays an essential safety role, and its integrity must be ensured during a variety of transient loading conditions. These can include off-normal conditions such as a pressurized thermal shock (PTS), as well as transients encountered during normal startup, shutdown, and testing of the reactor. Exposure to irradiation and elevated temperatures embrittles the RPV's steel over time, making it increasingly susceptible to failure due to propagation of fractures that could initiate at the locations of flaws introduced during the manufacturing process. As long-term operation scenarios are being considered for LWRs in the United States, it is important to have a flexible simulation tool that can be used to perform probabilistic evaluations of RPV integrity under a wide variety of conditions and incorporate improved predictive models of RPV steel embrittlement. The Grizzly code is being developed to meet these needs. This paper describes Grizzly's modular architecture, provides results of benchmarking studies of various components of Grizzly, and demonstrates the application of Grizzly on a model that includes plume effects that are difficult to represent in other codes being used in current practice.
\end{abstract}

\section{Introduction}

Nuclear light water reactors (LWRs) currently supply approximately $20 \%$ of the electric power generated in the United States [1]. As this fleet of reactors ages, many of the systems, structures, and components that comprise these facilities experience age-related degradation due to a variety of phenomena, some of which are specific to nuclear environments, but many of which are due to environmental conditions observed in a variety of civil and industrial facilities. To ensure the long-term sustainability of the nuclear generating capability in the United States, the U.S. Department of Energy's Light Water Reactor Sustainability (LWRS) program is supporting research to improve the understanding of these aging mechanisms. As part of this effort, the Grizzly code is being developed as a platform to simulate the progression of aging mechanisms in LWR systems, components, and structures, and to assess their ability to safely perform their intended engineering functions after being subjected to aging.

The reactor pressure vessel (RPV) plays an essential role in ensuring the safety of an LWR plant during both normal and off-normal conditions. It serves a structural role in supporting and containing the reactor core, and as part of the primary coolant loop serves as one of the barriers (which also include the nuclear fuel cladding and secondary containment system) to the release of fission products to the environment. RPVs are massive structures, ap- 
proximately $12 \mathrm{~m}$ high, $2 \mathrm{~m}$ in radius, and $0.2 \mathrm{~m}$ thick in the case of a pressurized water reactor (PWR). They can contain a potentially large number of flaws introduced during the manufacturing process. These flaws generally do not pose a concern during normal operation, but during a transient event, there is a potential safety concern that the elevated stresses during that event may be sufficient to result in the initiation and propagation of fracture at one of those flaws. Pressurized thermal shock (PTS) scenarios are among the most aggressive of these transient events because the RPV is simultaneously subjected to a rapid cool-down and internal pressure.

Over time, as it is exposed to fast neutrons and elevated temperatures, the ferritic steel that the RPV is composed of becomes increasingly brittle, which makes it more susceptible to fracture. The steel is temperature-dependent, behaving in a more brittle manner at low temperatures and in a ductile manner at higher temperatures. During a PTS event, at the same time that the material in an embrittled RPV is subjected to elevated stresses due to thermal gradients and internal pressures, its toughness is decreased due to the combined effects of embrittlement and being at a lower temperature. In making decisions regarding long term operation of an existing reactor, the ability of the RPV to withstand postulated transient events with sufficient safety margin given the projected progression of embrittlement over time must be ensured.

In prioritizing research needs for Grizzly development, initial emphasis has been placed on RPV and concrete structure degradation [2] because issues with either could potentially limit the life of a nuclear power plant due to the extreme difficulty of their replacement.

The problem of assessing RPV fracture under long term operation conditions spans multiple length and time scales. There is abundant data on the progression of RPV steel embrittlement spanning the lifetime of the existing reactor fleet taken from surveillance coupons exposed to the same conditions as the reactor, and embrittlement models (e.g. [3]) based on this data are valid for irradiation times up to the time span of that data. As part of the Grizzly effort, models are being developed at the atomistic and grain scales to improve confidence in predictions of the progression of RPV steel embrittlement under long-term exposure to the reactor environmental conditions over multiple decades. Performing an engineering evaluation of an RPV under transient conditions requires evaluating its global thermo-mechanical response to transient events that occur over the course of tens or hundreds of minutes, and performing engineering fracture mechanics evaluations of many individual flaws with length scales ranging from the order a millimeter to tens of millimeters.

There are currently multiple codes that can perform probabilistic fracture mechanics (PFM) analysis of RPVs. A survey by [4] assessed the relative capabilities of sev- eral codes. Of the codes surveyed, PASCAL [5] and FAVOR $[6,7]$ are the most mature and widely used, and can be considered the state of the art. These codes focus on the beltline region of the RPV, using a one-dimensional (1D) finite element model to represent its global thermomechanical response assuming that the RPV behaves as an infinite cylinder. Fracture analysis in these codes is performed using influence coefficient techniques, which permit accurate and extremely rapid computation of stress intensity factors based on through-wall stress profiles and coefficients obtained from detailed analysis. This is essential, because the Monte Carlo techniques employed to evaluate the probability of failure given a population of flaws evaluate the fracture parameters for a very large number of flaws, and performing a direct analysis of that many $3 \mathrm{D}$ flaw models would require an unfeasible amount of computational resources.

As part of the broader effort to develop tools for assessing integrity of a variety of LWR plant components, an engineering-scale PFM capability, which is described in this paper, is being developed in the Grizzly code. Similar to other codes such as FAVOR, Grizzly has facilities to compute the global thermo-mechanical response of an RPV under transient loading, and then to perform PFM analysis of a population of flaws using the stresses computed in the global thermo-mechanical analysis in two separate stages. Grizzly uses a tightly-coupled multiphysics, multidimensional finite element solver to compute the global RPV response. It employs Monte Carlo sampling and influence coefficient techniques for its PFM analysis. Grizzly currently only considers crack initiation based on linear elastic fracture mechanics, and does not yet consider several important phenomena, such as warm prestressing, crack propagation and arrest, and plastic effects, although its architecture will permit including these capabilities in the future.

The overarching goal of Grizzly PFM development is to provide a modern, flexible tool that can be used to make the material embrittlement research being conducted by the LWRS program available to end users for engineering analysis of RPVs. Some specific design aspects of Grizzly to that end that differentiate it from other codes are summarized below:

Modular Architecture A major driver for the development of Grizzly is the need to have a modern computational platform that can be flexible enough to accommodate a variety of models and modeling assumptions. Grizzly is based on the open-source MOOSE multiphysics finite element framework [8], which provides a set of interfaces that make it straightforward to develop applications that add physics model in a modular fashion. Grizzly follows that same modular philosophy in its design, employing an object-oriented modular architecture that read- 
ily permits the incorporation of new models for various parts of the RPV analysis problem.

Multidimensional Capability The existing state-of-the art codes permit the global RPV response to be represented only as that of an infinite cylinder using a 1D axisymmetric model. A variety of effects of interest can only be represented effectively using a higher-dimensional representation of the vessel. These include behavior in the nozzle region and coolant plume effects, which have been shown by [9] to be potentially significant. Grizzly is based on an inherently multidimensional finite element framework, and can represent the global RPV thermo-mechanical response using model dimensionality appropriate for the problem, and use results from that model in fracture evaluations.

General Flaw Geometry The reduced order representations currently used to compute fracture parameters on flaws are limited to consider only flaws aligned with the axes of the RPV. Recent experiences with the Belgian Doel 3 and Tihange 2 Nuclear Power Plants, where ultrasonic inspection revealed the presence of quasi-laminar flaws [10], has highlighted the need for a capability to simulate off-axis flaws. This is not the focus of the present paper, but Grizzly has comprehensive capabilities for evaluation of fracture integrals on flaws with arbitrary geometry.

High Performance Computing Two or three dimensional (2D or 3D) evaluation of the global RPV response can be computationally expensive, as is also the case for Monte Carlo simulations involve large numbers of random realizations of flaw populations. Being based on MOOSE, Grizzly provides access to parallel computation capabilities to greatly accelerate these computations using modern multiprocessor workstations and high performance computing platforms, both for computing the global RPV response to a transient as well as for PFM calculations.

This paper describes the architecture and workflow of the Grizzly code, demonstrates benchmarking of various aspects of Grizzly against reference solutions, and demonstrates the application of Grizzly to a problem where coolant plume effects are considered, which requires a multidimensional capability.

\section{Problem Summary and Analysis Components}

Before describing the details of the Grizzly implementation, it is useful to provide a high-level summary of the procedure for PFM analysis that is common to multiple PFM codes, and is also used by Grizzly. This procedure can be broken into three main phases that are performed sequentially:

\section{Global RPV thermo-mechanical response}

2. Probabilistic fracture analysis

3. Post-processing of probabilistic results

A key assumption in this process is that individual flaws have a negligible effect on the global thermo-mechanical response of the RPV, so that it can be modeled assuming that there are no flaws present. Once the stress and temperature fields from the global RPV model are available, they can be used as inputs for fracture evaluation at individual flaws. The results from these individual flaw analyses are then aggregated to compute statistical quantities of interest for a population of flaws.

\subsection{Global Thermo-Mechanical Response}

During a transient event, the stresses in an RPV are driven by both the effects of coolant temperature and pressure. As the vessel is flooded with coolant during a PTS event, the inner surface is rapidly cooled, leading to tensile stresses on the vessel interior due to thermal contraction of the material, which are balanced by compressive stresses on the vessel exterior. Pressurization of the vessel causes tensile stresses through the wall of the vessel.

Both stress and temperature fields are necessary for fracture evaluation. Because the deformation of the vessel is relatively small, it has little effect on the thermal solution, so this can be treated as a one-way coupling problem, where temperature fields are first computed, and then used as inputs for a mechanical solution.

Thermal response of the RPV is governed by the following form of the heat equation, which includes terms due to transient and conductive behavior, and neglects volumetric heating:

$$
\rho C_{p} \frac{\partial T}{\partial t}+\nabla \cdot q=0
$$

where $\rho$ is the density, $C_{p}$ is the specific heat, $T$ is the temperature, $t$ is time, and the heat flux $q$ is

$$
q=-k \nabla T
$$

where $k$ is the material's thermal conductivity. Both $k$ and $C_{p}$ vary as functions of temperature.

The mechanical response is computed to meet the equilibrium condition, where body forces can be neglected:

$$
\nabla \cdot \sigma=0
$$

where $\sigma$ is the Cauchy stress tensor. Small strain assumptions are valid, so the strains $\varepsilon$ can be computed as a function of the displacement vector $\boldsymbol{u}$ :

$$
\varepsilon=\frac{1}{2}\left(\nabla \boldsymbol{u}+(\nabla \boldsymbol{u})^{T}\right)
$$


A linear elastic constitutive model can be employed to compute the stress:

$$
\sigma=E:\left(\varepsilon-\varepsilon_{t h}\right)
$$

where $\boldsymbol{E}$ is the elasticity tensor and $\varepsilon_{\boldsymbol{t}}$ is a tensor containing the thermally induced strains. The coefficient of thermal expansion driving the thermally-induced strains varies with temperature, and must be properly accounted for according to the procedures described in [11].

The finite element method is typically employed as a numerical discretization technique to solve these partial differential equations. The RPV could be represented in 1D, $2 \mathrm{D}$, or $3 \mathrm{D}$, but a $1 \mathrm{D}$ axisymmetric representation is commonly used for computational efficiency and simplicity. If a 1D axisymmetric representation is employed, it is important to properly represent the mechanical deformation in the axial direction. A generalized plane strain condition should be employed, which constrains planar sections to remain planar, but allows for free expansion. In addition, a pressure to balance the applied coolant pressure must be applied in the axial direction.

\subsection{Reduced-Order Models for Fracture Analysis}

Once the global RPV response is computed, the computed stresses and temperatures can be used to evaluate the likelihood of fracture initiation and arrest. Deterministic or probabilistic procedures can be employed. Because the nature of the flaw population and the local material properties are uncertain, a probabilistic approach to evaluate the likelihood of fracture is warranted, and is used in the present effort.

In a probabilistic approach to evaluate the flaw population in an RPV, a large number of random realizations of potential flaw populations may be needed, which requires an efficient procedure for evaluating fracture parameters at flaws. Linear elastic fracture mechanics is typically employed, which is based on the deterministic calculation of the mode- $I$ stress intensity factor $K_{I}$. In general, numerically evaluating $K_{I}$ for a given flaw configuration requires running a detailed $2 \mathrm{D}$ or $3 \mathrm{D}$ finite element analysis of that flaw and evaluating fracture integrals. Performing such analyses for each individual flaw in a probabilistic evaluation of the flaw population would be prohibitively computationally expensive. Instead, these calculations are typically performed using the influence coefficient procedure originally proposed by [12], which takes advantage of the fact that contributions to $K_{I}$ from the individual components of a polynomial expansion of the far-field stress applied to a crack can be linearly superimposed.

In this technique, the through-wall distribution of the stress component normal to the crack $\sigma$ as a function of the depth $a^{\prime}$ relative to the flaw depth $a$ is described by a cubic polynomial:

$$
\sigma\left(\frac{a^{\prime}}{a}\right)=C_{0}+C_{1}\left(\frac{a^{\prime}}{a}\right)+C_{2}\left(\frac{a^{\prime}}{a}\right)^{2}+C 3\left(\frac{a^{\prime}}{a}\right)^{3}
$$

where the $C_{i}$ terms are the polynomial coefficients of the stress distribution. $K_{I}$ can then be expressed as:

$$
K_{I}=\sum_{i=0}^{3} C_{i} K_{i} \sqrt{\pi a}
$$

where $K_{i}$ are stress intensity factor influence coefficients (SIFICs). A given SIFIC $K_{i}$ can be computed by evaluating $K_{I}$ when applying the stress expansion of Equation 6 with all polynomial coefficients set to 0 except for $C_{i}$. These SIFICs can be evaluated for specific flaw geometries, and a variety of techniques can be used to evaluate them within a parameter space [13]. This approach is adopted by the ASME Boiler \& Pressure Vessel Code, which provides formulas for computing SIFICs for surface-breaking and subsurface (embedded) flaws in Section XI, Article A-3000 [14]. It should be noted that the polynomial stress expansion can be expressed either in terms of depth relative to flaw depth, as in Equation 6 or the total wall thickness if an adjustment is made to the SIFICs.

An important consideration for RPVs is that for surfacebreaking flaws, the stresses in the cladding can differ significantly from the base-metal stresses due to differences in the thermal expansion coefficients of those materials, and should be considered in the calculation of $K_{I}$. The principle of superposition can again be applied to compute $K_{I}$ as the summation of the contributions of the base metal $K_{I_{\text {base }}}$ and cladding $K_{I_{\text {clad }}}$ :

$$
K_{I}=K_{I_{\text {base }}}+K_{I_{\text {clad }}}
$$

This technique is employed by FAVOR, which uses a set of SIFICs for cladding that have been developed from detailed finite element models of various flaw geometries. The stress coefficients used in these calculations reflect the difference between the cladding and extrapolated base metal stresses.

The residual stresses that remain after post-welding heat treatment can have an important influence on the stress intensity factor for flaws in weld regions. These can be included by superimposing residual stresses on those due to the thermo-mechanical response to a transient loading. FAVOR uses residual stress distributions obtained by performing finite element simulations of experiments that measured the deformation of slots cut in experimental weld specimens. This feature is not yet implemented in Grizzly, but including this effect in the same manner is straightforward. Alternatively, residual stresses could be 
represented in Grizzly by directly simulating steps of the welding process in a 3D global RPV model prior to subjecting it to a transient loading.

\subsection{Embrittlement Model}

RPV embrittlement occurs due to the effects of damage to the crystal structure by fast neutrons and by the formation of precipitates, both of which serve to harden the material and decrease its fracture toughness. Modeling the progression of the fundamental underlying phenomena and their effects on the microstructure, and then accounting for their effects on engineering properties is a complex multiscale problem. For PFM analyses, it is essential to have a model that can be rapidly evaluated to permit the analysis of a large number of flaw realizations. The EONY model $[3,15]$ is a widely accepted physically motivated model that is calibrated to an extensive database of testing results, and is used in the FAVOR code. The embrittlement is represented by the shift in the reference nil-ductility transition temperature $R T_{\mathrm{NDT}}$.

Because of the shielding effects of the RPV steel, the fast neutron fluence varies through the RPV wall thickness, with significantly higher values (and hence, significantly higher embrittlement) on the inner surface of the RPV. In the absence of detailed neutron transport analysis, the attenuation law of [16] provides a simple exponential equation for the through-wall fluence decay that can be readily used for this purpose.

\subsection{Probabilistic Fracture Analysis}

A probabilistic fracture analysis can be performed on either a single flaw or on a collection of flaws. For a single flaw, the temperature-dependent Weibull statistical model for the conditional probability of fracture initiation (CPI) employed by FAVOR can be expressed as:

$$
\operatorname{CPI}\left(K_{I}\right)=1-\exp \left(-\left[\frac{K_{I}-a_{K_{I C}}}{b_{K_{I C}}}\right]^{c_{K_{I C}}}\right)
$$

if $K_{I}>a_{K_{I C}}$, otherwise CPI $=0$. The $a, b$, and $c$ coefficients in this equation are temperature-dependent:

$$
\begin{aligned}
a_{K_{I C}}\left(\Delta T_{r e l}\right) & =21.26+9.159 \exp \left(0.0406 \Delta T_{r e l}\right) \\
b_{K_{I C}}\left(\Delta T_{r e l}\right) & =17.15+55.09 \exp \left(0.0144 \Delta T_{r e l}\right) \\
c_{K_{I C}} & =4.0
\end{aligned}
$$

$\Delta T_{r e l}$ is $T-R T_{\mathrm{NDT}}$, where $T$ is the current temperature. $K_{I}, a_{K_{I C}}$, and $b_{K_{I C}}$ are all in units of $\mathrm{MPa} \sqrt{\mathrm{m}}$, and temperatures are in ${ }^{\circ} \mathrm{C}$.

Figure 1 graphically illustrates the dependence of CPI on temperature and $K_{I}$. As $K_{I}$ increases or the temperature decreases, CPI increases. Because temperature and
Figure 1: Conditional probability of initiation (CPI) as a function of temperature and $K_{I}$

$K_{I}$ vary over time, CPI varies over time. During a given transient event, the probability of fracture initiation at a given flaw is the maximum value of CPI over that transient.

A given RPV contains a population of many flaws, and ultimately, the quantity of interest is the aggregate CPI, or $\mathrm{CPI}_{\mathrm{RPV}}$ for initiation of fracture at any of the flaws given the occurrence of a given transient, which is computed as:

$$
\mathrm{CPI}_{\mathrm{RPV}}=1-\prod_{i=1}^{n_{\text {flaw }}}\left(1-\mathrm{CPI}_{i}\right)
$$

where $n_{\text {flaw }}$ is the number of flaws in the RPV, and $\mathrm{CPI}_{i}$ is the maximum CPI for flaw $i$ during that transient.

The set of postulated transients that could occur during off-normal conditions can vary widely in their likelihood of occurrence. Severe transients that cause high $\mathrm{CPI}_{\mathrm{RPV}}$ often have low probabilities of occurrence. Computing an overall probability of failure of an RPV involves taking the sum of the products of $\mathrm{CPI}_{\mathrm{RPV}}$ and the likelihood of occurrence of each transient.

\subsection{Uncertainty Quantification}

In practice, there is uncertainty in the geometric characteristics of the flaw population, in the material properties that affect fracture toughness, and in the neutron fluence. Random sampling can be used to quantify the mean value and uncertainty of CPI for a given RPV and transient.

FAVOR employs a two-level Monte Carlo sampling scheme for this purpose. At the outer level, a series of realizations of RPVs are generated. Each RPV realization consists of samples of global variables, steel chemistry parameters that apply globally to the major regions of the RPV, and a random flaw population whose geometric characteristics are defined by probability distributions. 
A different number of flaws is generated for each RPV realization. At the inner level, steel chemistry parameters are generated for each of the flaws in an RPV realization. These are local perturbations to the global properties of the major region (plate, weld, fusion zone) in which they are located.

Given these randomly generated parameters for the set of flaws, the local $\mathrm{RT}_{\mathrm{NDT}}$ for each flaw is computed and CPI can be computed for each flaw. Each RPV realization represents a defensible potential state of the RPV, and CPI is computed for each such realization. A sufficiently large number of RPV realizations is evaluated to compute the mean and standard deviation of CPI. In a post-processing phase, these are multiplied by the probabilities of occurrence of the transients.

It should be noted that the conditional probability of failure (CPF) can also be computed in a similar manner to $\mathrm{CPI}$. CPF is the probability of fracture propagating through the vessel, conditional on occurrence of the given transient loading. CPF accounts for the fact that some flaws can self-arrest when they reach a zone of decreased tensile stress, and is always equal to or lower than CPI. Grizzly does not yet have a crack propagation and arrest capability, so it does not currently compute CPF.

The procedures used by FAVOR to sample the parameters describing populations of flaws in the RPV realizations are provided below for flaw geometry (Section 2.5.1), chemistry (Section 2.5.2), and unirradiated RT $\mathrm{NDT}_{\mathrm{ND}}$ (Section 2.5.3).

\subsubsection{Flaw Geometry Sampling}

The geometric parameters sampled for individual flaws include the inner and outer depth of the flaw, the aspect ratio, and the flaw orientation. These are generated by FAVOR using files that define a set of distributions of flaw density, depth, and aspect ratio. These files are generated using the VFLAW tool developed by Pacific Northwest National Laboratory as part of the NRC's PTS Re-Evaluation Project [17], which are based on data described in [18].

These flaw population characterization files contain a set of 1000 tables of data, each of which is a unique distribution of the flaw population. These distributions define the density of flaws of a given through-thickness dimension, and for each dimension bin also define the distribution of the aspect ratio of flaws having that dimension. In a PFM analysis, the first 1000 RPV realizations use the first 1000 flaw distributions sequentially, after which the flaw distributions are re-used in sequence.

Three separate files are used to define distributions of embedded flaws in plates and welds, and surface breaking flaws (which can be in either plate or weld regions). The generated flaws can be oriented in either the axial or circumferential directions. In plate regions, the em- bedded flaws have equal probabilities of having axial or circumferential orientations. In weld regions, embedded flaws are oriented to align with the weld orientation. Inner surface-breaking flaws are all assumed to be in the circumferential orientation because of the process of applying the cladding, while outer surface-breaking flaw orientations are determined in the same manner as described above for embedded flaws.

\subsubsection{Chemistry Sampling}

The content of the alloying elements $\mathrm{Cu}, \mathrm{Ni}, \mathrm{Mn}$ and $\mathrm{P}$ is sampled, and the sampled contents are used as input in the EONY embrittlement model.By convention, sampled variables are indicated with a $\hat{\bullet}$ symbol and the $\leftarrow$ symbol indicates sampling from a distribution with the set of parameters defining that distribution specified in parentheses.

The user provides the following input:

- For each subregion: best estimates in weight $\%$ of the chemistry content: $\mathrm{Cu}_{\text {subr }}, \mathrm{Ni}_{\text {subr }}, \mathrm{Mn}_{\text {subr }}, \mathrm{P}_{\text {subr }}$

- Standard deviations of normal distributions for weld chemistry content: $\sigma_{\mathrm{Cu}, \mathrm{RPV}}, \sigma_{\mathrm{Ni}, \mathrm{RPV}}, \sigma_{\mathrm{P}, \mathrm{RPV}}$

- Standard deviations of normal distributions for plate chemistry content: $\sigma_{\mathrm{Cu}, \mathrm{RPV}}, \sigma_{\mathrm{Ni}, \mathrm{RPV}}, \sigma_{\mathrm{P}, \mathrm{RPV}}$

For each RPV realization, the global standard deviation of $\mathrm{Mn}$ (in wt\%) is sampled. For plates:

$$
\widehat{\sigma}_{\mathrm{Mn}, \mathrm{RPV}} \leftarrow \operatorname{Weibull}(0,0.06933,2.4708)
$$

for forgings:

$$
\widehat{\sigma}_{\mathrm{Mn}, \mathrm{RPV}} \leftarrow \mathrm{JSB}(0.00163,0.03681,0.83358,1.15153)
$$

where JSB is the JohnsonSB distribution. The global standard deviations of $\mathrm{Cu}, \mathrm{Ni}$, and $\mathrm{P}$ are assumed to be constant, so they are not sampled.

For each subregion of the RPV realization, the global subregion chemistry content is sampled. For plate and forging subregions, the content is sampled from normal distributions (denoted as $\mathcal{N}()$ ) from user-defined input:

$$
\begin{gathered}
\widehat{\mathrm{Cu}}_{\text {subr }} \leftarrow \mathcal{N}\left(\mathrm{Cu}_{\text {subr }}, \sigma_{\mathrm{Cu}, \mathrm{RPV}}\right) \\
\widehat{\mathrm{Ni}}_{\text {subr }} \leftarrow \mathcal{N}\left(\mathrm{Ni}_{\text {subr }}, \sigma_{\mathrm{Ni}, \mathrm{RPV}}\right) \\
\widehat{\mathrm{Mn}}_{\text {subr }} \leftarrow \mathcal{N}\left(\mathrm{Mn}_{\text {subr }}, \widehat{\sigma}_{\mathrm{Mn}, \mathrm{RPV}}\right) \\
\widehat{\mathrm{P}}_{\text {subr }} \leftarrow \mathcal{N}\left(\mathrm{P}_{\text {subr }}, \sigma_{\mathrm{P}, \mathrm{RPV}}\right)
\end{gathered}
$$

For weld subregions, the standard deviations of the normal distributions for $\mathrm{Cu}, \mathrm{Ni}$ and $\mathrm{Mn}$ are sampled from specific normal distributions. The subregion chemistry content is then sampled from normal distributions defined by 
the best estimate means and the sampled standard deviations.

$$
\begin{aligned}
\hat{\sigma}_{\mathrm{Cu}} & \leftarrow \mathcal{N}\left(0.167 \times \mathrm{Cu}_{\mathrm{subr}}, \min \left(0.0718 \times \mathrm{Cu}_{\mathrm{subr}}, 0.0185\right)\right) \\
\hat{\sigma}_{\mathrm{Ni}} & \leftarrow \mathcal{N}(0.029,0.0165) \\
\hat{\sigma}_{\mathrm{Mn}} & \leftarrow \operatorname{Weibull}(0.01733,0.04237,1.83723)
\end{aligned}
$$

$$
\begin{aligned}
\widehat{\mathrm{Cu}}_{\text {subr }} & \leftarrow \mathcal{N}\left(\mathrm{Cu}_{\text {subr }}, \widehat{\sigma}_{\mathrm{Cu}, \text { subr }}\right) \\
\widehat{\mathrm{Ni}}_{\text {subr }} & \leftarrow \mathcal{N}\left(\mathrm{Ni}_{\text {subr }}, \widehat{\sigma}_{\mathrm{Ni}, \text { subr }}\right) \\
\widehat{\mathrm{Mn}}_{\text {subr }} & \leftarrow \mathcal{N}\left(\mathrm{Mn}_{\text {subr }}, \hat{\sigma}_{\mathrm{Mn}, \text { subr }}\right) \\
\widehat{\mathrm{P}}_{\text {subr }} & \leftarrow \mathcal{N}\left(\mathrm{P}_{\text {subr }}, \widehat{\sigma}_{\mathrm{P}, \text { subr }}\right)
\end{aligned}
$$

For each flaw, the local chemistry is calculated as the sum of the global chemistry and a small local variability. $\mathrm{Mn}$ has a larger local variability and is sampled from a normal distribution.

$$
\begin{aligned}
\widehat{\mathrm{Cu}}_{\text {flaw }} & =\widehat{\mathrm{Cu}}_{\text {subr }}+\widehat{\Delta}_{\mathrm{Cu}} \\
\widehat{\mathrm{Ni}}_{\text {flaw }} & =\widehat{\mathrm{Ni}}_{\text {subr }}+\widehat{\Delta}_{\mathrm{Ni}} \\
\widehat{\mathrm{Mn}}_{\text {flaw }} & =\mathcal{N}\left(\widehat{\mathrm{Mn}}_{\text {subr }}, \widehat{\sigma}_{\mathrm{Mn}}\right) \\
\widehat{\mathrm{P}}_{\text {flaw }} & =\widehat{\mathrm{P}}_{\text {subr }}+\widehat{\Delta}_{\mathrm{P}}
\end{aligned}
$$

For plates, the local variability is calculated by:

$$
\begin{aligned}
\widehat{\Delta}_{\mathrm{Cu}} & \leftarrow \operatorname{Logistic}\left(-3.89 \times 10^{-7}, 0.00191\right) \\
\widehat{\Delta}_{\mathrm{Ni}} & \leftarrow \operatorname{Logistic}\left(-1.39 \times 10^{-7}, 0.00678\right) \\
\widehat{\Delta}_{\mathrm{P}} & \leftarrow \operatorname{Logistic}\left(1.3 \times 10^{-5}, 0.000286\right) \\
\widehat{\sigma}_{\mathrm{Mn}} & \leftarrow \operatorname{JSB}(0.00163,0.03681,0.83358,1.15153)
\end{aligned}
$$

For welds, the local variability is calculated by:

$$
\begin{aligned}
\widehat{\Delta}_{\mathrm{Cu}} & \leftarrow \operatorname{Logistic}\left(6.85 \times 10^{-8}, 0.0072\right) \\
\widehat{\Delta}_{\mathrm{Ni}} & \leftarrow \operatorname{Logistic}(-0.0014,0.00647) \\
\widehat{\Delta}_{\mathrm{P}} & \leftarrow \operatorname{Logistic}\left(3.27 \times 10^{-6}, 0.000449\right) \\
\widehat{\sigma}_{\mathrm{Mn}} & \leftarrow \operatorname{JSB}(0.00163,0.03681,0.83358,1.15153)
\end{aligned}
$$

\subsubsection{RT $_{\text {NDT }}$ Sampling}

The transition temperature $\mathrm{RT}_{\mathrm{NDT}}$ characterizes the irradiation-induced embrittlement of the RPV steel. The shift in $\mathrm{RT}_{\mathrm{NDT}}, \Delta \mathrm{RT}_{\mathrm{NDT}}$, compared to the unirradiated value, $\mathrm{RT}_{\mathrm{NDT}(0)}$, is estimated using the EONY model based on the sampled chemistry content of the steel and the neutron fluence at the depth of the flaw. $\mathrm{RT}_{\mathrm{NDT}}$ for each flaw is calculated using the formula:

$$
R T_{\mathrm{NDT}}=\widehat{R T}_{\mathrm{NDT}(0)}-\widehat{R T}_{\mathrm{epistemic}}+\widehat{\Delta R T}_{\mathrm{NDT}}
$$

The epistemic uncertainty, $\mathrm{RT}_{\text {epistemic }}$ is included to account for the difference between values of $\mathrm{RT}_{\mathrm{NDT}}$ and $\mathrm{T}_{0}$ estimated directly from fracture toughness data using the Master Curve method [6]. The magnitude of $\mathrm{RT}_{\text {epistemic }}$ is sampled once for each subregion of an RPV realization from a distribution given by:

$$
\widehat{R T}_{\text {epistemic }}=\text { Weibull }(-29.5,78.0,1.73)
$$

For each flaw, $\widehat{R T}_{\mathrm{NDT}(0)}$ is sampled from a normal distribution defined by the mean $\mathrm{RT}_{\mathrm{NDT}(0)}$ and standard deviation $\sigma_{R T_{\mathrm{NDT}(0)}}$ of the subregion (input by the user).

$$
\widehat{R T}_{\mathrm{NDT}(0)}=\mathcal{N}\left(R T_{\mathrm{NDT}(0)}, \sigma_{R T_{\mathrm{NDT}(0)}}\right)
$$

\section{Grizzly implementation Details}

\subsection{High Level Workflow}

The goal of the development of engineering-scale PFM capabilities in Grizzly is to provide the same capabilities offered by current state-of-the-art PFM codes in a modern modular computational framework that readily permits including higher-dimensional effects, use on multiprocessor computers, and incorporation of embrittlement models being developed by the LWRS program. As such, the overall workflow in Grizzly is very similar to that described above. The FAVOR code served as a template for the Grizzly PFM algorithm design, and results from Grizzly models should be able to closely match FAVOR results. This has been ensured during the development process by benchmarking individual components of Grizzly against their FAVOR counterparts.

As with other PFM codes, a Grizzly RPV analysis consists of two phases: a global thermo-mechanical analysis of the RPV to a given transient, and a PFM analysis that can either consider a set of flaws with prescribed parameters, or a set of randomly sampled RPV realizations from which statistics are computed. These two phases are both performed using the same Grizzly executable, but a different set of modules are activated in these phases.

\subsection{Global Thermo-Mechanical Analysis}

To compute the global RPV transient response, Grizzly does not impose restrictions on the dimensionality of the model employed, but allows the user to create a 1D, 2D, or 3D finite element mesh to represent the RPV geometry. The MOOSE framework was designed to support arbitrary model dimensionality. An extensive capability for modeling general coupled thermal and mechanical physics has been developed in the common physics modules that are included with the open-source MOOSE framework. MOOSE's development was originally largely motivated by the needs of the Bison fuel performance code [19], 
which is also based on MOOSE, and Grizzly makes use of these common capabilities that are used by multiple codes.

The standard way to solve coupled physics models in MOOSE is to solve for all field variables in a tightly coupled manner by solving a single system of equations that contains all variables. These can alternatively be solved in a loosely coupled fashion. The relative merits of these two approaches were evaluated in [20] for multiple classes of problems, including the RPV global response. It was shown that because the RPV problem involves essentially one-way coupling between the thermal and mechanical models, it can be solved slightly more efficiently using loose coupling. This is in contrast to other problems that involve stronger two-way feedback between those models, which are solved significantly more efficiently using tight coupling. Tight coupling is the more straightforward of these approaches to use in MOOSE, and it is employed in standard Grizzly global RPV response calculations for convenience rather than efficiency.

Figure 2 shows snapshots of the stress contours for a representative RPV subjected to a PTS transient represented using full 3D, 2D planar, and 1D axisymmetric representations in Grizzly at the same point in time. The 1D and 2D models represent the response of a vessel that extends infinitely in the axial direction, and closely reproduce the behavior of the global model in the beltline region. The 2D planar and 1D axisymmetric models both employ generalized plane strain conditions to allow axial expansion and contraction, but force planar sections to remain planar. The lower dimensional models require significantly lower computational resources than the $3 \mathrm{D}$ model, and are appropriate for investigating many scenarios of interest.

The beltline region of the RPV in these models has an inner radius of $2.197 \mathrm{~m}$. The total wall thickness is 21.92 $\mathrm{cm}$, including a $4.064 \mathrm{~mm}$ thick layer of stainless steel cladding. The PTS transient used for this simulation applies the representative global temperature and pressure histories shown in Figure 7 which are used later in this paper for benchmarking PFM calculations. Temperaturedependent functions for the coefficient of thermal expansion of the base metal and cladding used in the FAVOR example problem suite are used here. These are mean thermal expansion functions with a reference temperature of $21.1^{\circ} \mathrm{C}$. A stress-free temperature of $253.3^{\circ} \mathrm{C}$ is assumed, and the initial coolant temperature in the transient is $286.0^{\circ} \mathrm{C}$.

\subsection{Reduced Order Models for Fracture Analysis}

Grizzly makes use of the influence coefficient approach summarized in Section 2.2 to compute time histories of $K_{I}$ for embedded and surface-breaking flaws. For both

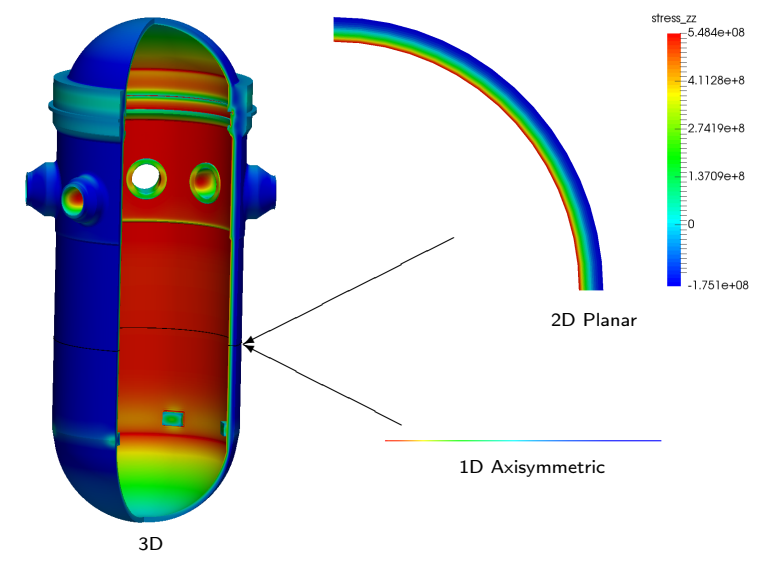

Figure 2: Results of 1D axisymmetric, 2D planar, and 3D Grizzly models of the global response of a PWR RPV at a point in time during a PTS event. $1 \mathrm{D}$ and $2 \mathrm{D}$ models represent the response on the plane in the beltline region shown by the dark line in the $3 \mathrm{D}$ model, and match the behavior of the $3 \mathrm{D}$ model on that plane.

axial and circumferential surface-breaking flaws, the procedure of Section XI, Article A-3000 of the ASME Boiler \& Pressure Vessel Code [14] is used to compute SIFICs. This includes the updates documented in [21,22] for axial flaws that first appeared in the 2017 version of the code. These are used in conjunction with the cladding SIFICs used by FAVOR Version 16.1 to account for the effect of stresses in the cladding. It should be noted that FAVOR 16.1 does not directly use the A-3000 SIFICs for all cases pending verification of these relatively new models against benchmark solutions. Grizzly can directly use the A-3000 SIFICs for all surface-breaking flaws, and it also provides a compatibility option to use the same SIFICs as FAVOR 16.1 for most flaw geometries.

For embedded flaws, Grizzly uses the procedure of FAVOR 16.1 documented in [6]. This is based on superposition of membrane and bending components of the stress, and uses the procedure developed by [23] to analytically compute the membrane stress correction factors in Section XI, Article A-3000 of the 2013 and earlier versions of the ASME Boiler \& Pressure Vessel Code [24]. It should be noted that the 2015 and 2017 versions of that code [25] employ a new procedure for subsurface (embedded) flaws based on influence coefficients proposed in [26] that has not yet been implemented in Grizzly, but will be in future versions. The older method currently used by Grizzly and FAVOR has been found to include significant conservatism, so there is motivation to use this new procedure in the future.

To compute the time-varying coefficients to the polynomial expansion of the stress field, Grizzly provides two 
options. For 1D simulations in which there is no axial or azimuthal variation in the stress field, the stress coefficients are evaluated during the global model evaluation by sampling the stress field at multiple points through the thickness of both the cladding and base metal of the RPV. A least-squares procedure is used to fit these to polynomials of user-defined order, and the time histories of the coefficients to those polynomials are written out to commaseparated value (CSV) text files. The temperature fields are output in the same manner. The polynomial coefficients are then read in during the PFM analysis, and either used directly to evaluate $K_{I}$ using Equation 7, or used to sample the polynomial to evaluate needed stress or temperature fields at specific locations.

Alternatively, for higher-dimensional simulations in which these fields potentially vary axially or azimuthally, the full finite element solution is written out to a mesh file, typically using the ExodusII format [27]. During the PFM phase of the analysis, the solution is read in from this file. For a given flaw, a line of points is constructed extending radially through the RPV wall at the flaw's azimuthal and axial position. The needed solution quantity is sampled at those points, and least-squares fitting is used to construct a polynomial of the desired order to represent the radial variation of that quantity. It is important to note that the fracture calculation in these scenarios considers throughwall variation in stress fields at the flaw location, but does not consider higher-order effects due to axial or azimuthal variations in those fields.

The SIFIC procedure is referred to here as a reducedorder approach because $K_{I}$ is computed from a small set of input parameters (the coefficients to the polynomial representation of the through-wall variation of the stress field) Because it is based on a finite element framework, Grizzly also provides the ability to directly solve for the stress fields around a flaw using a detailed 2D or 3D finite element model. The flaw geometry can be represented either by incorporating it in the mesh topology [28], or using the extended finite element method (XFEM) [29, 30] to represent it as a mesh-independent discontinuity. For such an analysis, the detailed fracture model can be constructed to represent the material in the vicinity of the flaw. A given transient can be directly simulated by prescribing boundary conditions from the solution on the global RPV model, or this capability can be used to develop SIFICs for configurations not represented in the standard solutions.

Fracture analysis results for a representative flaw during a PTS transient using both an SIFIC approach and an XFEM simulation in Grizzly, compared with a reference FAVOR solution are shown in Figure 3. This compares the time history of $K_{I}$ at the deepest point of a circumferentially oriented circular surface-breaking flaw with a depth of $2.197 \mathrm{~cm}$ in the RPV in Figure 2 subjected to the PTS transient in Figure 7. Grizzly SIFIC results are shown for
Figure 3: Comparison of time history of $K_{I}$ during the representative PTS transient shown in Figure 7 for an axisaligned circumferential surface-breaking flaw in the RPV shown in Figure 2. Grizzly SIFIC results use through-wall stress solutions computed from 1D, 2D, and 3D Grizzly global RPV models. These are compared with results from a 3D Grizzly XFEM model and a FAVOR model of the same flaw.

cases where stresses are computed from global 1D axisymmetric, 2D planar and full 3D RPV models. The 1D and 2D models are the same models shown in Figure 2, while the 3D model is an extrusion of that $2 \mathrm{D}$ mesh, to provide a $90^{\circ}$, quarter-symmetry representation of a ring of the beltline region. The XFEM model uses that same $90^{\circ} 3 \mathrm{D}$ ring mesh, but it is highly refined in the local region of the flaw. In the XFEM model, $K_{I}$ is computed using an interaction integral at points along the tip of the flaw, which is represented using XFEM. There is good agreement between all these results.

\subsection{Embrittlement Model}

To compute the embrittlement, Grizzly employs the EONY model [3, 15], which is one of the models offered by FAVOR. Through-wall fluence variation is computed using the procedure of [16], which is also used by FAVOR.

\subsection{Probabilistic Fracture Analysis}

Grizzly implements the procedure described in Section 2.4 to perform PFM analysis of either individual flaws or populations of flaws. For the latter case, Monte Carlo sampling is used to generate a set of realizations of parameters describing the population of flaws in an RPV. Grizzly closely follows the procedures of FAVOR as outlined in Section 2.5 for generating the geometry, chemistry, and unirradiated $R T_{\mathrm{NDT}}$ parameters describing random realizations of the flaw population. 
The only difference in Grizzly's flaw population generation relative to FAVOR is due to the fact that Grizzly can consider spatial variation in the stress and temperature fields when performing PFM modeling based on 2D or 3D models of the global RPV response. In addition to the set of parameters describing flaw geometry used by FAVOR (inner and outer depth, aspect ratio, and orientation), Grizzly also generates variables describing the axial and azimuthal positions of the flaws. These parameters are used to determine the spatial locations of the flaws for evaluating local stresses and temperatures. Flaws are assumed to be uniformly distributed axially and azimuthally within forgings and plates, and are uniformly positioned along the weld fusion lines in weld zones.

\subsection{Grizzly Architecture for PFM}

The MOOSE framework and the applications based on it, such as Grizzly, are typically used to solve partial differential equations (PDEs), and the pluggable interfaces MOOSE provides to implement physics models are generally geared to such applications. However, MOOSE can be run in a mode where it does not solve a PDE, but merely steps through time. MOOSE also provides pluggable interfaces for general-purpose models that are not necessarily related to finite element modeling. The Grizzly PFM capability was developed based on MOOSE, and makes use of a variety of general MOOSE features to perform operations such as reading solutions from meshes.

Like MOOSE, Grizzly is written in the $\mathrm{C}++$ language and has an object-oriented design. A set of base classes that define the interfaces needed for the PFM functionality are designed to have derived classes inherit from them to provide specializations of the components for specific purposes. Figure 4 shows a diagram of the set of base classes used to define Grizzly's PFM functionality and their interdependencies. In the block-formatted Grizzly input file, the user defines which specializations of each of these objects are used, along with their associated parameters. For a PFM analysis, the user must specify all of the classes shown in Figure 4. This system is very flexible in how these objects can be used together, and makes it very straightforward to implement new models that provide portions of this functionality. A deterministic analysis can be performed using only a subset of these classes (KICalculator and the classes that it depends on).

A brief summary of these classes and the specializations of their functionality provided via derived classes is provided in Table 1. Note that by convention, the name Base is appended to the names of the classes in Figure 4 for abstract base classes for which functionality must be provided by a derived class. There are several utility classes in addition to those listed in Table 1 that query these objects to report statistics on the aggregate CPI from a set of

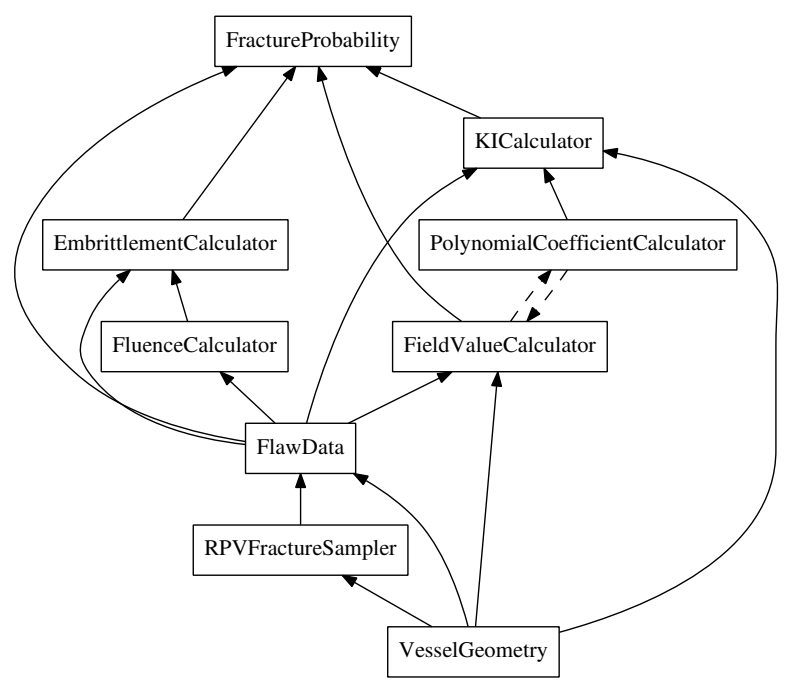

Figure 4: Dependency diagram of the classes used to provide Grizzly's PFM functionality. The dashed arrows indicate an optional dependency because only some of the classes deriving from the indicated classes have that dependency.

samples, or report results for individual flaws.

\subsection{Parallel Implementation and Algorith- mic Flow}

There are a number of available probabilistic analysis tools that can be used to generate random samples and drive arbitrary codes to evaluate physics models using those samples, and perform statistical analysis on the results. Early iterations of the Grizzly design attempted to use this approach. However it became evident that because the PFM problem involves very large numbers of rapidly evaluable samples, repeatedly calling Grizzly from a probabilistic driver code to evaluate a single flaw results in significant overhead, and hinders the efficiency of the analysis.

To avoid this overhead, the current design of Grizzly generates the samples and processes the full set of samples within a single instance of a Grizzly application. The full set of data defining each flaw is stored in memory, and the analysis loops through time, evaluating the full set of flaws for each time step. This requires that a sufficient amount of memory is available to store this data, but has the advantage that it allows for each step of the global RPV model results to be loaded only once, which avoids extra computation. Figure 5 shows a flowchart of Grizzly's PFM process, including the loops through time steps and flaws.

Evaluating a large number of flaws can be a timeconsuming operation, and is a task that is readily performed in parallel. This belongs to the class of problems 
Table 1: Descriptions of classes shown in Figure 4 that provide Grizzly's PFM functionality. Inherited classes are indented and shown below their respective base classes.

\begin{tabular}{|c|c|}
\hline VesselGeometry & $\begin{array}{l}\text { Stores basic information about the RPV geometry, and provides ac- } \\
\text { cess to that information to a variety of classes that need that data. }\end{array}$ \\
\hline RPVFractureSampler & $\begin{array}{l}\text { Generates the set of RPV realizations including the geometric and } \\
\text { chemistry variables associated with each flaw using the procedure de- } \\
\text { scribed in Sections } 2.5 .1 \text { and 2.5.2. }\end{array}$ \\
\hline FlawDataBase & Stores the parameters for a population of flaws or a single flaw. \\
\hline SingleFlawData & Stores user-defined parameters for a single flaw \\
\hline FlawDataFromSampler & Stores randomly generated samples from RPVFractureSampler \\
\hline FlawDataFromFile & $\begin{array}{l}\text { Stores data on a flaw population read from a CSV file, which could } \\
\text { have been generated from sampling in a previous run. }\end{array}$ \\
\hline FieldValueCalculatorBase & Computes the value of a field variable at a requested location. \\
\hline FieldValueFromCoefficients & Samples a polynomial using its coefficients to provide the field value. \\
\hline FieldValueFromExodus & Obtains an interpolated field value from an ExodusII mesh. \\
\hline FieldValueFromFile & Reads a field value directly from a CSV file. \\
\hline EmbrittlementCalculatorBase & Computes $\mathrm{RT}_{\mathrm{NDT}}$ \\
\hline EmbrittlementEONY & Computes $\mathrm{RT}_{\mathrm{NDT}}$ using the EONY model. \\
\hline FluenceCalculatorBase & Computes the fluence at any position in the vessel. \\
\hline FluenceAttenuatedFromSurface & $\begin{array}{l}\text { Implements a simple exponential decay function from a value pre- } \\
\text { scribed at the inner wetted surface for a given subregion. }\end{array}$ \\
\hline KICalculatorBase & $\begin{array}{l}\text { Computes } K_{I} \text { for the full set of flaws currently being considered for } \\
\text { each time step. }\end{array}$ \\
\hline KIAxisAlignedROM & $\begin{array}{l}\text { Uses SIFICs to compute this for axis-aligned surface-breaking and } \\
\text { embedded flaws. This creates a set of lightweight objects that com- } \\
\text { pute } K_{I} \text { for the various types of flaws, and also creates and uses in- } \\
\text { terchangeable objects for computing the SIFICs, allowing different } \\
\text { models to be easily implemented and used. }\end{array}$ \\
\hline UserDefinedROM & $\begin{array}{l}\text { Computes } K_{I} \text { using a user-defined set of SIFICs for a specific flaw } \\
\text { geometry }\end{array}$ \\
\hline KIFromFile & $\begin{array}{l}\text { Directly reads a time history of } K_{I} \text { for a single flaw from a CSV file } \\
\text { for use in a PFM calculation. This is primarily useful for debugging } \\
\text { purposes. }\end{array}$ \\
\hline FractureProbability & $\begin{array}{l}\text { Computes CPI and the maximum CPI for each flaw being considered } \\
\text { over the course of the transient using information computed by the } \\
\text { other classes. }\end{array}$ \\
\hline
\end{tabular}

commonly described as "embarrassingly parallel" [31] because the individual flaw evaluations can be performed independently, and the only required communication between processors is in computing the aggregate CPI for the set of flaws in all RPV realizations, which may be evaluated on different processors. To perform this evaluation in parallel, Grizzly generates a subset of the flaw population on each processor, evaluates CPI for that set of flaws on each processor, and performs a parallel reduction to compute the aggregate CPI for each RPV realization. The flaw generation is performed using the same random number stream regardless of the number of processors used, so that an identical set of parameter samples is generated from a given seed and the resulting CPI computations are independent of the number of processors.
Figure 6 shows the parallel speedup as a function of processor count for two Grizzly PFM calculations. This scalability study was performed using up to 8 computing nodes of a large cluster, each with 218 -core processors. This was run both for a simulation where the global response is obtained by directly reading stress coefficients from a 1D calculation, and for a case where the global response is obtained from the mesh of a $2 \mathrm{D}$ simulation. The 2D case involves much more computational effort per flaw evaluation because it involves looking up field values from the mesh to compute stress coefficients. Both cases show good parallel scalability with lower numbers of processors, but the speedup begins to degrade when the communication costs begin to be significant relative to the costs of individual flaw calculations. Because the 


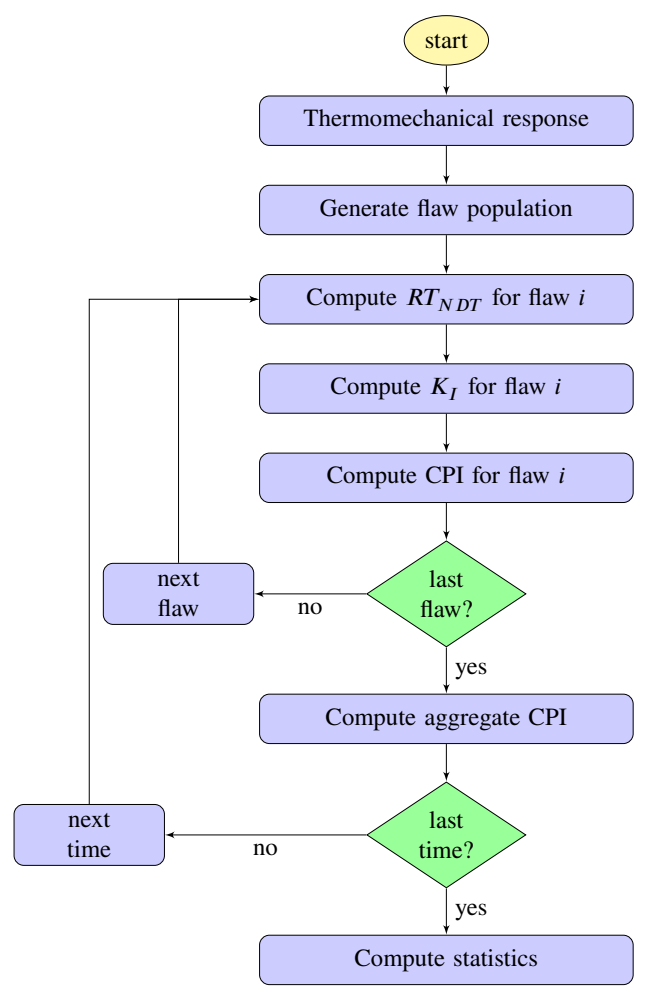

Figure 5: Algorithmic flow of Grizzly PFM analyses. This is unique for a PFM code because the outer loop is over time steps rather than flaws.
Figure 6: Parallel scalability of Grizzly probabilistic evaluations of large sets of RPV realizations. Speedup is the ratio of the single processor wall clock time to the multi-processor wall clock time, which is ideally equal to the number of processors used. Results are shown for 100000 realizations of a single plate in an RPV with a total of 17406700 flaws with global results obtained from a 1D simulation, and for 1000 realizations with a total of 174067 flaws with global results from a 2D simulation.

flaw evaluations are much more computationally expensive for the 2D case, its parallel scalability is closer to the ideal scenario with higher processor counts. This parallel evaluation capability provides significant practical advantages, allowing for rapid evaluation of a very large number of RPV realizations, which is often necessary for convergence of the Monte Carlo simulations. The simulations presented in this paper took advantage of this capability, and the $2 \mathrm{D}$ and $3 \mathrm{D}$ cases were run on up to 360 processors.

\section{Benchmarking}

In the process of Grizzly development, the major components of the code were benchmarked by comparison with FAVOR to ensure the algorithms were correctly implemented. These include the capabilities for modeling the global RPV response, deterministic and probabilistic fracture of a single flaw, and random sampling. Once these individual components were benchmarked, PFM results on randomly generated flaw populations were compared against FAVOR equivalents. Such a comparison is more involved than a comparison of individual components because the randomly generated samples will not be the same between the two codes, but with a sufficient number of RPV realizations, the average CPI computed by the two codes should be close if they are implementing the same sampling strategies and deterministic algorithms.

For benchmarking purposes, a particularly aggressive 
Figure 7: Temperature and pressure histories applied to RPV for benchmarking simulations and plume effect demonstrations. The "Global Temperature" is a temperature applied uniformly to the inner surface of an RPV when a spatially uniform temperature is assumed. The "Plume Temperature" is used here to represent the temperature history applied in a plume region. The "Plume Temperature" is a notional approximation of the more rapid cooling that would occur near an inlet.

PTS transient history with a low frequency of occurrence from a FAVOR benchmark problem was applied to a PWR RPV with prototypical dimensions. Figure 7 shows these histories. To test the global RPV response and deterministic $K_{I}$ calculations, time histories of stress for a number of through-thickness locations and $K_{I}$ for a variety of flaw geometries were compared, and found to match very closely. A representative comparison under this transient for one specific flaw geometry was shown in Figure 3. Results from a set of benchmarking calculations for a wide range of specific axis-aligned flaw geometries is available in [30]. A time-varying heat transfer coefficient between the coolant and vessel can be specified, although for simplicity, a constant value of $56.8 \mathrm{~kW} /\left(\mathrm{m}^{2} \mathrm{~K}\right)$ was used in all analyses in this paper.

A set of benchmarking analyses were performed using a single plate region within an RPV represented using 1D, 2D, and 3D models of the global RPV thermo-mechanical response. Figure 8 shows the $2 \mathrm{D}$ and $3 \mathrm{D}$ models used to represent the global RPV response in the region of interest. The plate of interest, shown as a darker region in the models in Figure 8, spans $80^{\circ}$, and has a height of 0.30 $\mathrm{m}$. The RPV inner radius $(2.197 \mathrm{~m})$, cladding thickness $(4.064 \mathrm{~mm})$, and total wall thickness $(21.92 \mathrm{~cm})$ are the same as those used previously for the results shown in Figures 2 and 3.

The 2D model uses a generalized plane strain represen-
Figure 8: 2D (top) and 3D (bottom) models used to compute global RPV response for benchmarking response of a single $80^{\circ}$ plate positioned at the center of the $90^{\circ}$ quartersymmetry models with FAVOR under uniform loading conditions. These models are also used for demonstrating modeling of an idealized coolant plume effect by applying a more rapidly-decreasing temperature history to a $10^{\circ}$ wide portion of the panel shown in blue in these models. The red dots in these figures indicate the locations of the set of flaws in a single random realization of the flaw population, and the line of white dots passing through each red dot indicates the set of points where the stress was sampled from the global model for computing stress coefficients used in the $K_{I}$ calculation. 
tation for the out-of-plane response. The 3D model imposes a fixed displacement boundary condition on the bottom surface in the vertical direction, and imposes a constraint that the vertical displacement be equal across the top surface. The 1D model (shown in Figure 2) consists of a single row of line elements and imposes axisymmetric conditions for the computation of the hoop strains. The strains in the axial direction are uniform across the model and are computed to ensure that the area integral of the axial stress is equal to the force applied that axial direction by the internal pressure in the RPV. This is a $1 \mathrm{D}$ version of the generalized plane strain condition used in the 2D model. All models use 2nd-order finite elements (Quad9 and Hex27 for 2D and 3D models, respectively), with strong biasing to have more elements near the inner surface.

The same seed was used to generate the random samples used in the 1D, 2D, and 3D benchmark models for uniform conditions, so the sampled flaw populations were identical in all cases. Azimuthal and axial positions are generated for all flaws, but these are only used where applicable in higher-dimensional models (both are used for 3D models, only the azimuthal position is used for 2D planar models, and neither are used for 1D models). These models all assume that the RPV has been subjected to 40 effective full power years, and exposed to a uniform neutron fluence of $4.0 \times 10^{19} / \mathrm{cm}^{2}$ on the inner surface, with a coefficient of variation (COV) of 0.118 for sampling fluence for a given plate region, and a $\mathrm{COV}$ of 0.056 for sampling fluence for individual flaws. For steel chemistry sampling, mean values for the single subregion of $\mathrm{Cu}, \mathrm{Ni}, \mathrm{Mn}$, and $\mathrm{P}$ content (weight \%) are specified as $\mathrm{Cu}_{\text {subr }}=0.2, \mathrm{Ni}_{\text {subr }}=0.6$, $\mathrm{Mn}_{\text {subr }}=1.3$, and $\mathrm{P}_{\text {subr }}=0.01$, and the standard deviations of the normal distributions used to sample $\mathrm{Cu}, \mathrm{Ni}$, and $\mathrm{P}$ for plates are $\sigma_{\mathrm{Cu}, \mathrm{RPV}}=0.0073, \sigma_{\mathrm{Ni}, \mathrm{RPV}}=0.0244$, and $\sigma_{\mathrm{P}, \mathrm{RPV}}=0.0013$. The initial unirradiated nil-ductility reference temperature, $R T_{\mathrm{NDT} 0}$ is $10.0^{\circ} \mathrm{C}$, and the standard deviation of that quantity is $9.44^{\circ} \mathrm{C}$.

The mean values for the chemistry content and $R T_{\mathrm{NDT} 0}$ are representative of values for plates as tabulated in the FAVOR theory manual and in the Reactor Vessel Integrity Database [32]. The values used for the COV of fluence and standard deviation of $R T_{\mathrm{NDT} 0}$ are representative values from FAVOR examples, and the standard deviations for the chemistry variables are recommended values from FAVOR. It should be noted that the parameters used were intentionally chosen to cause relatively high embrittlement within the representative regime to facilitate benchmarking with a reasonable number of Monte Carlo iterations. Cases with low failure probability have increased scatter in the results, requiring more Monte Carlo iterations to demonstrate convergence.

To benchmark the Grizzly implementation of the random sampling scheme, histograms of the individual sampled variables were compared against those generated from FAVOR using the same set of parameters for this plate. Populations of both embedded and surfacebreaking flaws have been generated, and Grizzly identically matched the number of flaws in FAVOR. The examples presented here focus on the population of embedded flaws. Figure 9 shows comparisons of distributions of some of the sampled variables between Grizzly and FAVOR for the embedded flaw population for 100000 realizations of this single-plate RPV model.

Overall, there is very good agreement between the samples generated by Grizzly and FAVOR. The sampling procedure for the Mn concentration involves using sampling one distribution to obtain parameters for another distribution, and has the largest discrepancy between Grizzly and FAVOR of any of the chemistry variables. A minor discrepancy between the codes was found in the sampled inner flaw tip depth. FAVOR uniformly distributes the flaws separately over the inner and outer half of the RPV, but uses the midpoint of the total RPV thickness, rather than of the base metal, for the dividing point between those regions. This results in a slightly increased flaw density on the inner half, and a corresponding decreased density in the outer half. For cool-down transients, this is conservative, and results in a small increase in the computed CPI. Grizzly's sampling procedure distributes flaws uniformly through the base metal. To make the two codes as consistent as possible for benchmarking, a minor adjustment was made to FAVOR to match Grizzly's behavior, and the FAVOR results presented here use that adjustment.

Once the global RPV response, $K_{I}$ solutions, and sampled variables have been favorably compared between Grizzly and FAVOR, the two codes can be compared on a full PFM calculation with a large number of RPV realizations of the same RPV. Because Grizzly and FAVOR use different random number generators, their random flaw realizations should differ, and accordingly, the Monte Carlo convergence histories should also differ, but the mean CPI computed by the two codes should converge to the same value with a sufficiently large number of RPV realizations. Figure 10 shows the convergence history of the mean CPI of the single plate with the embedded flaw population considered here for the Grizzly models (1D, 2D, and 3D) compared with FAVOR. There is significant variation in the computed CPI between RPV realizations, especially for cases with low CPI. Because of this, the mean CPI changes significantly early in the Monte Carlo iteration history.

It is clear that a large number of RPV realizations must be run before the Monte Carlo simulation can be considered converged. After 100000 RPV realizations, the mean CPI values computed by Grizzly are $3.27 \times 10^{-5}$, $3.27 \times 10^{-5}$, and $3.28 \times 10^{-5}$, for the $1 \mathrm{D}, 2 \mathrm{D}$, and $3 \mathrm{D}$ models, respectively, while the corresponding FAVOR result is $3.44 \times 10^{-5}$ (approximately 5\% difference between Griz- 
Figure 10: Comparison of mean CPI as a function of the number of RPV realizations between Grizzly models (1D, 2D, and 3D) and FAVOR. The FAVOR 1D and Grizzly 1D models were run to 500000 realizations to demonstrate convergence, while the Grizzly 2D and 3D models were run to 100000 iterations.

zly 1D and FAVOR). To show that Grizzly and FAVOR eventually converge to the same solution with enough iterations, the 1D Grizzly model and FAVOR model were both run to 500000 iterations, at which point CPI from the 1D Grizzly model is $3.30 \times 10^{-5}$, while the FAVOR counterpart is $3.34 \times 10^{-5}$ (approximately $1 \%$ difference). All other models shown in the remainder of this paper use 100000 RPV realizations.

The 2D and 3D Grizzly models should compute nearly identical results to the 1D Grizzly model because of the uniform loading conditions. The discrepancies between those models are very minor, and are attributable to subtle differences in the finite element discretizations and the ways that fields are interpolated and sampled. This example provides evidence that Grizzly implements the FAVOR algorithms for CPI calculation, and that those procedures work consistently for 1D, 2D, and 3D models of the global RPV response.

Figure 9: Comparisons of histograms of representative sampled variables over 100000 random realizations of the single plate used for benchmarking between Grizzly and FAVOR, showing (from top to bottom) flaw size, flaw aspect ratio, depth to inner flaw tip, $\mathrm{Cu}$ concentration, $\mathrm{Mn}$ concentration, and $R T_{\mathrm{NDT} 0}$
The computational costs of the PFM analysis increase significantly going from $1 \mathrm{D}$ to $2 \mathrm{D}$ and $3 \mathrm{D}$ models because of the effort involved in extracting field values from a large mesh database. For 100000 RPV realizations, the 1D simulations took about 1 minute on 18 processors, while the 2D simulation required about 2 hours on 360 processors and the 3D simulation took about 20 hours on 360 processors. There are multiple ways the algorithm could be optimized for improved efficiency with higher dimensional models that will be considered in future development. 


\section{Probabilistic Fracture Mechan- ics Demonstration on Higher- Dimensional Model}

To demonstrate Grizzly's ability to perform PFM calculations considering local nonuniformities in the RPV response, the same RPV models used in the benchmarking study of the previous section have been modified to demonstrate how coolant plume effects can be considered on a population of flaws. The same transient used previously for the uniform cases is applied to the plate, except that the notional plume temperature history shown in Figure 7 is applied to a $10^{\circ}$ wide region at the center of the plate (shown in blue in Figure 8) intended to represent a region near the inlet. It has been shown in [9] that the CPI for individual flaws can increase by orders of magnitude in the presence of plume effects.

It is important to emphasize here that this analysis is intended only to demonstrate Grizzly's capability to include plume effects, and does not represent an actual PTS scenario, which would require a detailed 3D fluid dynamics analysis to provide a realistic temperature distribution in the plume region. The temperature applied to the plume region is $100^{\circ} \mathrm{C}$ lower than the global temperature during the initial cooling phase, until it reaches the minimum temperature in the global history, at which point it remains constant until the temperature rises, when it tracks the global temperature. It is also important to note that the plume effects will be most pronounced immediately adjacent to an inlet, which is above the beltline region of the RPV that experiences the highest irradiation. The mechanical boundary conditions and irradiation conditions used the uniform case in the previous section were assumed here, which likely result in increased mechanical constraint and embrittlement relative to actual conditions.

The 2D and 3D global models of the RPV panel shown in Figure 8 were both run with the plume temperature history applied to a local region. Figure 11 shows profiles of the temperature and hoop stress fields at one point during the transient for the 3D model. The $2 \mathrm{D}$ results are not shown, but they are similar to the fields on the cutaway midplane surface on the top of the 3D models shown. The plume temperature history clearly results in significantly elevated stresses and decreased temperatures in its vicinity, both of which tend to increase susceptibility to fracture.

The PFM procedure was run using the same 100000 RPV realizations that were used in the uniform cases, but using the global response from the 2D and 3D plume models. Figure 12 shows a scatter plot of the spatial location of all flaws with nonzero CPI in all RPV realizations, colored by CPI. For comparison purposes, the results from the 2D benchmark case in the previous section (which are nearly 
Figure 13: Scatter plots showing flaws with nonzero CPI plotted in terms of relative temperature and $K_{I}$ at the point in time when maximum CPI is reached. These are shown for the 3D uniform loading case (top) and 3D plume case (bottom).

Figure 12: Scatter plots showing flaw tip positions for all flaws in all 100000 RPV realizations with nonzero CPI in terms of depth from inner wetted surface to flaw tip and azimuthal coordinate, color coded by maximum CPI. Results are shown for the 2D model with uniform temperature (top), the 2D plume model (middle), and the 3D plume model (bottom).

identical to those of the uniform 3D case) are shown. As expected, the flaws closer to the inner surface have significantly higher CPI, and there is little azimuthal variation. In the $2 \mathrm{D}$ and $3 \mathrm{D}$ plume cases, there is a significant increase in the number of flaws with nonzero CPI in the plume region. Both the density of flaws with high CPI and the depth of the region containing flaws with high CPI are markedly increased. This effect is more pronounced in the 2D model than the 3D model because it represents the plume region as extending infinitely in the axial direction, so the top and bottom of the panel considered have experience more aggressive stress and thermal conditions than they do in the 3D model.

Figure 13 shows similar scatter plots for the 3D models with uniform and plume conditions, but using coordinates of relative temperature and $K_{I}$ at the point in time when flaws reached their maximum CPI. There is clearly a significant increase in the number of flaws with high CPI across a broad range of temperature and $K_{I}$ conditions in the plume case relative to the uniform case.

After $100000 \mathrm{RPV}$ realizations, the 2D model predicted a mean CPI of $1.03 \times 10^{-3}$, while the equivalent for the $3 \mathrm{D}$ model was $2.86 \times 10^{-4}$. Based on the previous discussion, the higher CPI for the 2D model is expected, and it is clear that using reduced dimensionality representations of the global response with local nonuniformities can introduce excess conservatism. Both of these results represent CPI values significantly higher than the baseline uniform CPI of $3.28 \times 10^{-5}$ (for the 3D Grizzly model). Although this is not intended to represent an actual RPV, this does indicate that this warrants further investigation with more representative conditions.

\section{Summary}

The Grizzly code is being developed to address a variety of aging mechanisms in LWR components, including RPVs, which are a high priority research focus. Grizzly provides a modern, flexible architecture that makes it easily extensible, able to address effects that can only be considered with a higher-dimensional code, and able to take advantage of multi-processor computers. This allows Grizzly to serve as a tool to enable fundamental material embrittlement research being performed under the LWRS program 
to be used in engineering analyses of the effects of RPV embrittlement.

An overview of Grizzly's modular architecture and algorithmic flow have been provided, along with a summary of benchmarking against FAVOR, which represents the current state of the art code in this area. The local effects of a coolant plume on the probabilistic response of a portion of an RPV containing a population of flaws has been evaluated with this tool using higher-dimensional representations of the global response of the RPV to the transient to demonstrate its applicability to such problems. These results with contrived conditions clearly indicate that this effect warrants further investigation with more realistic models of actual RPV geometry and plume conditions.

Grizzly is still under active development, and currently provides a fully usable, although limited PFM analysis capability. It is still missing important features such as the ability to consider warm prestressing effects, compute the conditional probability of failure considering crack propagation and arrest, and assess multiple transients in a single calculation, but these are readily permitted by Grizzly's code architecture, and will be incorporated in the future.

\section{Acknowledgments}

The support of the FAVOR team (Paul Williams, B. Richard Bass, Terry Dickson, and Hilda Klasky) in providing the FAVOR code and explaining its algorithms is gratefully acknowledged. Also, the support of Andrew Slaughter and Cody Permann of the MOOSE team for Grizzly's use of the MOOSE framework is gratefully acknowledged.

This work was funded by the US Department of Energy under the Light Water Reactor Sustainability (LWRS) program. The submitted manuscript has been authored by a contractor of the U.S. Government under Contract DEAC07-05ID14517. Accordingly, the U.S. Government retains a non-exclusive, royalty free license to publish or reproduce the published form of this contribution, or allow others to do so, for U.S. Government purposes.

\section{References}

\section{References}

[1] Electric Power Annual 2016. US Energy Information Administration, December 2017.

[2] Hai Huang and Benjamin W. Spencer. Grizzly model of fully coupled heat transfer, moisture diffusion, alkali-silica reaction and fracturing processes in concrete. In V. Saouma, J. Bolander, and E. Landis, editors, Proceedings of the 9th International Confer- ence on Fracture Mechanics in Concrete Structures (FraMCoS-9), number 194, Berkeley, CA, May 29June 12016.

[3] E.D. Eason, G.R. Odette, R.K. Nanstad, and T. Yamamoto. A physically-based correlation of irradiation-induced transition temperature shifts for RPV steels. Journal of Nuclear Materials, 433(13):240-254, February 2013.

[4] Guian Qian and Markus Niffenegger. Procedures, methods and computer codes for the probabilistic assessment of reactor pressure vessels subjected to pressurized thermal shocks. Nuclear Engineering and Design, 258:35-50, May 2013.

[5] Kunio Onizawa, Hiroyuki Nishikawa, and Hiroto Itoh. Development of probabilistic fracture mechanics analysis codes for reactor pressure vessels and piping considering welding residual stress. International Journal of Pressure Vessels and Piping, 87(1):2-10, January 2010.

[6] P.T. Williams, T.L. Dickson, B. R. Bass, and H. B. Klasky. Fracture Analysis of Vessels - Oak Ridge, FAVOR, v16.1, computer code: Theory and implementation of algorithms, methods, and correlations. Technical Report ORNL/LTR-2016/309, Oak Ridge National Laboratory, Oak Ridge, TN, September 2016 .

[7] T.L. Dickson, P. T. Williams, B. R. Bass, and H. B. Klasky. Fracture Analysis of Vessels - Oak Ridge, FAVOR, v16.1, computer code: User's guide. Technical Report ORNL/LTR-2016/310, Oak Ridge National Laboratory, Oak Ridge, TN, September 2016.

[8] Derek Gaston, Chris Newman, Glen Hansen, and Damien Lebrun-Grandié. MOOSE: A parallel computational framework for coupled systems of nonlinear equations. Nuclear Engineering and Design, 239(10):1768-1778, October 2009.

[9] Guian Qian, Markus Niffenegger, Medhat Sharabi, and Nathan Lafferty. Effect of non-uniform reactor cooling on fracture and constraint of a reactor pressure vessel. Fatigue \& Fracture of Engineering Materials \& Structures, March 2018.

[10] Valéry Lacroix and Pierre Dulieu. Structural integrity assessment of Doel 3 and Tihange 2 RPVs affected by hydrogen flakes: Methodology and results. page V01AT01A058. ASME, July 2016.

[11] Markus Niffenegger and Klaus Reichlin. The proper use of thermal expansion coefficients in finite element calculations. Nuclear Engineering and Design, 243:356-359, February 2012. 
[12] H. F. Bückner. A novel principle for the computation of stress intensity factors. Z. angew Math. Mech., 50:529-546, 1970.

[13] William M. Hoffman, Matthew E. Riley, and Benjamin W. Spencer. Surrogate model development and validation for reliability analysis of reactor pressure vessels. In ASME Pressure Vessels and Piping Conference, number PVP2016-63341, Vancouver, BC, Canada, July 17-21 2016.

[14] ASME Boiler and Pressure Vessel Code, Section XI, Rules for Inservice Inspection of Nuclear Power Plant Components. Number ASME BPVC.XI-2017. ASME, 2017.

[15] E.D. Eason, G.R. Odette, R.K. Nanstad, and T. Yamamoto. A physically based correlation of irradiation-induced transition temperature shifts for rpv steels. Technical Report ORNL/TM-2006/530, Oak Ridge National Laboratory, 2006.

[16] Regulatory guide 1.99: Radiation embrittlement of reactor vessel materials, Revision 2, 1988.

[17] F. A. Simonen, S. R. Doctor, G. J. Schuster, and P. G. Heasler. A generalized procedure for generating flaw-related inputs for the FAVOR code. Technical Report NUREG/CR-6817, PNNL-14268, Pacific Northwest National Laboratory, Richland, WA, March 2004.

[18] G. J. Schuster, S. R. Doctor, A. F. Pardini, and S. L. Crawford. Characterization of flaws in U.S. reactor pressure vessels. Technical Report NUREG/CR6741, Vol. 2, PNNL-11143B, Pacific Northwest National Laboratory, Richland, WA, August 2000.

[19] R. L. Williamson, J. D. Hales, S. R. Novascone, M. R. Tonks, D. R. Gaston, C. J. Permann, D. Andrs, and R. C. Martineau. Multidimensional multiphysics simulation of nuclear fuel behavior. J. Nuclear Materials, 423:149-163, 2012.

[20] S.R. Novascone, B.W. Spencer, J.D. Hales, and R.L. Williamson. Evaluation of coupling approaches for thermomechanical simulations. Nuclear Engineering and Design, 295:910-921, August 2015.

[21] Steven X. Xu, Russell C. Cipolla, Darrell R. Lee, and Douglas A. Scarth. Improvements in Article A-3000 of Appendix A for Calculation of Stress Intensity Factor in Section XI of the 2015 Edition of ASME Boiler and Pressure Vessel Code. Journal of Pressure Vessel Technology, 139(1):010801, August 2016.
[22] Steven X. Xu, Darrell R. Lee, Douglas A. Scarth, and Russell C. Cipolla. Update on Stress Intensity Factor Influence Coefficients for Axial ID Surface Flaws in Cylinders for Appendix A of ASME Section XI. In Proceedings of the ASME 2015 Pressure Vessels and Piping Conference, number PVP201546009. ASME, July 2015.

[23] R. C. Cipolla. Computational method to perform the flaw evaluation procedure as specified in the asme code, section xi, appendix a. Technical Report EPRI Report NP-1181, Failure Analysis Associates, September 1979.

[24] ASME Boiler and Pressure Vessel Code, Section XI, Rules for Inservice Inspection of Nuclear Power Plant Components. ASME, 2013.

[25] ASME Boiler and Pressure Vessel Code, Section XI, Rules for Inservice Inspection of Nuclear Power Plant Components. Number ASME BPVC.XI-2015. ASME, 2015.

[26] Katsumasa Miyazaki, Fuminori Iwamatsu, Shin Nakanishi, and Masaki Shiratori. Stress Intensity Factor Solution for Subsurface Flaw Estimated by Influence Function Method. In ASME Pressure Vessels and Piping Conference, number PVP2006ICPVT-11-93138, Vancouver, BC, Canada, July 2327 2006. ASME.

[27] Larry A Schoof and Victor R Yarberry. EXODUS II: a finite element data model. Technical Report SAND92-2137, UC-705, Sandia National Laboratories, Albuquerque, NM, September 1994.

[28] Benjamin Spencer, Marie Backman, William Hoffman, and Pritam Chakraborty. Reactor pressure vessel integrity assessments with the Grizzly simulation code. In Transactions of SMiRT-23, Paper 710, Manchester, UK, August 10-14 2015.

[29] John Dolbow, Ziyu Zhang, Benjamin Spencer, and Wen Jiang. Fracture capabilities in Grizzly with the eXtended Finite Element Method (X-FEM). Technical Report INL/EXT-15-36752, Idaho National Laboratory, Idaho Falls, ID, September 2015.

[30] Benjamin W. Spencer, William M. Hoffman, and Wen Jiang. Enhancements to engineering-scale reactor pressure vessel fracture capabilities in Grizzly. Technical Report INL/EXT-17-43427, Idaho National Laboratory, September 2017.

[31] Ian Foster. Designing and building parallel programs, volume 78. Addison Wesley Publishing Company Boston, 1995. 
[32] US Nuclear Regulatory Commission. Reactor vessel integrity database version 2.0.1 (RVID 2), July 2000. 